Supporting information for

\title{
Multi-objective Optimization of TEG Dehydration Process for BTEX Emission Mitigation using Machine Learning and Metaheuristic Algorithms
}

\author{
Rajib Mukherjee ${ }^{1,2^{*}}$, Urmila M. Diwekar $^{2}$ \\ ${ }^{1}$ Department of Chemical Engineering, The University of Texas Permian Basin, Odessa, TX 79762, USA \\ ${ }^{2}$ Vishwamitra Research Institute, Crystal Lake, Illinois, 60012, USA
}

* To whom correspondence should be addressed: mukherjee_r@utpb.edu; ph. 432 5523457

The Supporting Information contains 6 pages, 1 Figure and 1 Table.

Table of content

1. Surrogate model generation with Support Vector Regression (SVR)

2. Selection of the range of the variables

Figure S1. Schematic diagram showing the classification

$$
\text { of data points using SVR }
$$

Table S1. Variables and range 


\section{Surrogate model generation with Support Vector Regression (SVR)}

We need to generate random realizations of the input variables to find the random response in the region of the sample space. The sample space should be surrounding the optimal design point in order to find the sensitivity of the system with respect to the variables. For a problem containing $p$ continuous input variables, the responses from the process systems under different conditions can be used in a real-value function estimation using SVR (1). If $X(n \times p)$ is the matrix of $n$ realizations (sample size) of $p$ input variables, then we can generate a $Y(n \times 1)$ vector of response. The $n$ observations in the training dataset can be represented as $D=\left\{\boldsymbol{x}_{i}, y_{i}\right\}_{i=1}^{n}$, where $\boldsymbol{x}_{i} \in \boldsymbol{X} \subseteq \mathbb{R}^{p}$ represent a vector in the input space of $p$ input variables

and $y_{i} \in \mathbb{R}$ represent the corresponding scalar output value. The objective of SVR is to find a regression function $y=f(\boldsymbol{x})$ that can predict the outputs $\{y\}$ for a new set of input-output data, $\{x, y\}$, drawn from the same joint probability $P\{x, y\}$ as the training data. The linear estimation function $f: \mathbf{X} \rightarrow \mathbb{R}$ in the transformed feature space $\mathcal{F}$ is given as:

$$
f(\boldsymbol{x})=\boldsymbol{w}^{\top} \Phi(\boldsymbol{x})+b
$$

Eq. 1

where $\Phi: \mathbf{X} \rightarrow \mathcal{F}$ is the nonlinear transformation from the input space $\boldsymbol{X} \subseteq \mathbb{R}^{p}$ to the high dimensional feature space $\mathcal{F} . \boldsymbol{w} \in \mathcal{F}$ is the coefficient vector and $b \in \mathbb{R}$ is a constant known as bias. In the feature space $\mathcal{F}$, linear regression is performed for predicting $y$. Thus, the problem of nonlinear regression in lower-dimensional input space $\mathbb{R}^{p}$ is transformed into a linear regression in the high-dimensional feature space $\mathcal{F}$. The unknown parameters $\boldsymbol{w}$ and $b$ are learned using the training set $D$ by minimizing the following objective which is the sum of regularization and empirical risk:

$$
\min _{\boldsymbol{w}, b} \frac{1}{2}\|\boldsymbol{w}\|_{2}^{2}+\sum_{i} C\left(y_{i}-f\left(\boldsymbol{x}_{i}\right)\right)
$$

Eq. 2

Where $\frac{1}{2}\|w\|_{2}^{2}$ represents regularization and $C($.$) is a cost function estimating the empirical$ loss given as (2):

$$
C(y-f(x))= \begin{cases}0, & \text { if }|y-f(x)| \leq \varepsilon, \\ |y-f(x)|-\varepsilon & \text { otherwise. }\end{cases}
$$


Eq. 3

This is called the $\varepsilon$-insensetive loss function where $\varepsilon$ is a precision parameter (should not be confused with constraint parament $\varepsilon_{i}$ of multi-objective optimization problem). Figure S1 is the graphical representation of SVR. $\varepsilon$ is the radius of the tube represented by a dashed line around the regression function represented by a solid line. Points outside the tube having positive and negative deviations are represented by slack variables $\xi$ and $\xi^{*}$, respectively. In SVR the estimation function $f(\boldsymbol{x})$ fits the data in a manner such that the training error is minimized by minimizing individual slack variables $\xi_{i}$ and $\xi_{i}{ }^{*}$, and $\|\boldsymbol{w}\|_{2}^{2}$.

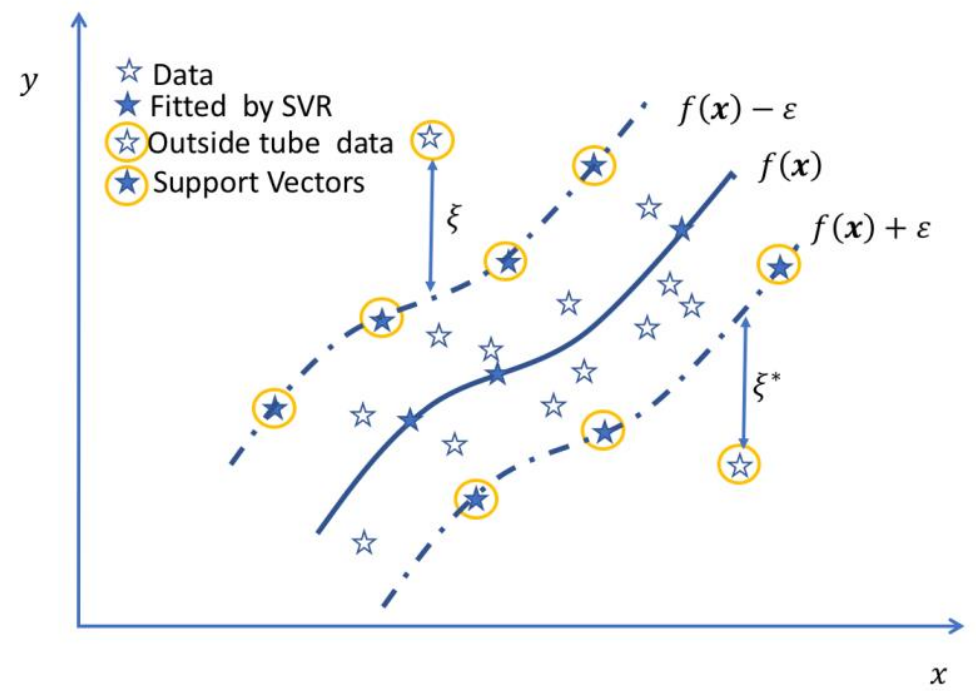

Figure S1. Schematic diagram showing the classification of data points using SVR

The Lagrangian dual of the optimization problem can be written as (3):

$$
\underset{\boldsymbol{\alpha}}{\max }-\frac{1}{2} \sum_{i, j}\left(\alpha_{i}^{*}-\alpha_{i}\right)\left(\alpha_{j}^{*}-\alpha_{j}\right) \boldsymbol{K}\left(\boldsymbol{x}_{i}, \boldsymbol{x}_{j}\right)-\varepsilon \sum_{i}\left(\alpha_{i}^{*}+\alpha_{i}\right)+\sum_{i} y_{i}\left(\alpha_{i}^{*}-\alpha_{i}\right)
$$

Eq. 4

Subject to:

$$
\begin{array}{rr}
\sum_{i}\left(\alpha_{i}^{*}-\alpha_{i}\right)=0 & \text { Eq. } 5 \\
0<\alpha_{i}, \alpha_{i}^{*}<C & \text { Eq. } 6
\end{array}
$$


Where $\alpha_{i}, \alpha_{i}^{*}$ are the Lagrange multipliers and $\boldsymbol{K}\left(\boldsymbol{x}, \boldsymbol{x}_{i}\right)$ is the reproducing kernel function given as (4):

$$
\boldsymbol{K}\left(\boldsymbol{x}_{i}, \boldsymbol{x}_{j}\right)=\Phi\left(\boldsymbol{x}_{i}\right)^{\top} \Phi\left(\boldsymbol{x}_{j}\right)
$$

Eq. 7

The corresponding $\boldsymbol{w}=\sum_{i}\left(\alpha_{i}-\alpha_{i}^{*}\right) \Phi\left(\boldsymbol{x}_{i}\right)$ and the estimation function $f: \mathbf{X} \rightarrow \mathbb{R}$ is represented as:

$$
f(\boldsymbol{x})=\sum_{i}\left(\alpha_{i}^{*}-\alpha_{i}\right) \boldsymbol{K}\left(\boldsymbol{x}, \boldsymbol{x}_{i}\right)+b .
$$

Eq. 8

There are several forms of the kernel function like radial bias function (RBF), $n^{\text {th }}$ degree polynomial $(5,6)$. Although RBF is frequently used as SVR models, in the present work, the $n^{\text {th }}$ degree polynomial, as shown in Equation 9 is found to be more accurate.

$$
K\left(x_{i}, x_{j}\right)=\left[1+\left(x_{i}, x_{j}\right)\right]^{n}
$$

Eq. 9

It is to be mentioned that out of $n$ training data, there are only $m$ instances when the data points are on the margins. These are called support vectors $(S V)$. For the support vectors, the Lagrangian multiplier $\left(\alpha_{i}^{*}-\alpha_{i}\right) \neq 0$. The $S V$ s can be thought of as the most informative data points that compress the information content of the training set.

The bias, $b$ is obtained using:

$$
b= \begin{cases}y_{i}-f\left(\boldsymbol{x}_{\boldsymbol{i}}\right)_{b=0}-\varepsilon & \text { for } \alpha_{i} \in(0, C), \\ y_{i}-f\left(\boldsymbol{x}_{\boldsymbol{i}}\right)_{b=0}+\varepsilon & \text { for } \alpha_{i}^{*} \in(0, C) .\end{cases}
$$

Eq. 10

\section{Selection of the range of the variables}

The range of variables as mentioned are based on literature. For a feed rate of 17.5 MMSCFD, the range of variables used as obtained from Braek et al. (7) are shown in the Table S1. It is to be mentioned that Braek et al. (7) has only selected the glycol circulation rate and the stripping gas flow rate as significant parameters for their optimization study. They have also 
modified flash tank pressure and the reboiler temperature in their work. The optimized value of the four parameters are also shown in Table S1.

Table S1: Variables with their range and optimal points by Braek et al.

\begin{tabular}{|l|l|l|l|l|}
\hline No. & Variable name & Unit & Range & $\begin{array}{l}\text { Optimal } \\
\text { point by } \\
\text { Braek et al. }\end{array}$ \\
\hline 1. & Glycol circulation rate & sgpm & $4.4-8.8$ & 5.28 \\
\hline 2. & Absorber pressure & psig & $300-1000$ & \\
\hline 3. & Inlet glycol Temperature & Fahrenheit & $140-212$ & \\
\hline 4. & Flash gas pressure & psig & $30-100$ & 87.02 \\
\hline 5. & Reboiler Temperature & Fahrenheit & $360-400$ & 380 \\
\hline 6. & Stripping gas rate & MSCFD & $50-300$ & 60.24 \\
\hline
\end{tabular}

Optimizing the process parameters in the TEG dehydration process, Braek et al 2001 were able to reduce the BTEX emission from 109.5 ton/yr to 53.29 ton/yr for a plant processing 17.5 MMSCFD of natural gas. Simulating the present process having a throughput of 100 MMSCFD at the optimal condition as found by Braek et al. (7) resulted in BTEX emission of 585.06 ton/yrs and dry gas water content of $3.89 \mathrm{lbm} / \mathrm{MMscf}$. It is to be mentioned that the BTEX emission depends on the natural gas throughput and gas composition. It is to be mentioned that the BTEX emission depends on the natural gas throughput and gas composition. Thus, it is imperative to obtain the optimal parametric values for a given process condition.

\section{References:}

1. Awad, M., \& Khanna, R. Support vector regression. In Efficient learning machines, 2015, pp. 6780 Apress, Berkeley, CA.

2. Vapnik, V. Statistical Learning Theory, Wiley, New York, 1998

3. Vapnik, V., Golowich, S., Smola, A. Support vector method for function approximation, regression estimation and signal processing, Adv. Neural Inform. Process. Syst., 1996, 9, 281287.

4. Minh, H. Q. Some properties of Gaussian reproducing kernel Hilbert spaces and their implications for function approximation and learning theory. Constructive Approximation, 2010, $32(2), \quad 307-338$. 
5. Nandi, S., Badhe, Y., Lonari, J., Sridevi, U., Rao, B. S., Tambe, S. S., \& Kulkarni, B. D. Hybrid process modeling and optimization strategies integrating neural networks/support vector regression and genetic algorithms: study of benzene isopropylation on Hbeta catalyst. Chemical Engineering Journal, 2004, 97(2-3), 115-129

6. Sivaramakrishnan, K., Nie, J., de Klerk, A., \& Prasad, V. (2018). Least squares-support vector regression for determining product concentrations in acid-catalyzed propylene oligomerization. Industrial \& Engineering Chemistry Research, 57(39), 13156-13176.

7. Braek, A. M., Almehaideb, R. A., Darwish, N., \& Hughes, R. Optimization of process parameters for glycol unit to mitigate the emission of BTEX/VOCs. Process Safety and Environmental Protection, 2001, 79(4), 218-232. 